\title{
ANTENATAL INVESTIGATION OF DUCTUS VENOSUS VELOCITY AS A METHOD OF DETECTING THE FETAL HEART FAILURE, CAUSED BY PARVOVIRUS B19 INFECTION
}

\author{
N. P. Bondarenko, A. V. Aksonova \\ BOGOMOLETS NATIONAL MEDICAL UNIVERSITY, KYIV, UKRAINE
}

Background. The article describes the methods and results of investigation of blood flow velocity waveforms in fetal ductus venosus (DV). These studies are used to visualize the degree of fetal heart failure and determine its further clinical course.

Objective. The study was aimed to predict the development of heart failure in the fetuses that were infected with parvovirus B19 infection during 11-14 gestation weeks by measuring the Doppler parameters of blood flow velocity in the DV.

Methods. Our investigation involved 20 pregnant women aged from 18 to 30 years old who were infected with parvovirus B19 infection during the period from 11 to 14 weeks of gestation. The DV was determined by means of color Doppler. Fetal echocardiography (EchoCG) was performed by means of the ultrasound scanner Philips HD IIXE device (USA) using a transabdominal convex probe with the frequency of $3.5 \mathrm{MHz}$, operating in a $C D C$ mode and the frequency filter at $100 \mathrm{~Hz}$. The A-wave directivity evaluation in the DV was investigated according to the Guideline Principles of the Fetal Medicine Foundation (www.fetalmedicine.com). Statistical processing of data was carried out using the package of applied programs Microsoft Office Excel 2016 and Statistica 6, Stata 12.

Results. In 16 of 20 (80\%) fetuses we did not observe any absent or reversed A-wave flow in the DV during atrial contraction as well as any fetal echocardiographic pathological signs. In 2 (10\%) cases a reversed A-wave flow in the DV in a combination with EchoCG-signs of overload of left side of heart, resulting in enlargement (dilatation) of left atrium and left ventricle were detected. In 2 (10\%) cases the presence of a reversed A-wave flow in the DV and EchoCG-signs of fetal heart failure (reduction of cardiac output, significant dilatation of left ventricle) were evidenced.

The results of the study confirm that with the expansion of fetal nuchal translucency thickness, the systolic blood flow velocity in the DV increases with the correlation coefficient $r=0.594$, which proves a linear dependence between these two ultrasonography parameters.

Conclusions. The linear correlation between the presence of a reversed A-wave blood flow velocity in the DV and the overload of left side of fetal heart, development of heart failure (20\% of the total number of examined women) were proved.

KEY WORDS: ductus venosus (DV); heart failure; parvovirus B19 infection; peak systolic blood flow velocity; fetal echocardiography (EchoCG).

\section{Introduction}

Parvovirus B19 infection is a potentially lifethreatening disease [1] for a fetus, especially during the first two trimesters of pregnancy that can cause a variety of signs of its damage (severe anemia, ventriculomegaly, hypertrophic myocardiopathy and pericardial effusion, nonimmune fetal hydrops, hydropic or nonhydropic intrauterine fetal death, intrauterine growth retardation, thrombocytopenia, meconium peritonitis, hepatic calcifications, abnormal longterm neurodevelopment, ascites, placento-

Corresponding author: Anastasiia Aksonova, Department of Obstetrics and gynaecology №1, National Bogomolets National Medical University, 13 T. Shevchenko, Kyiv, Ukraine, 03150

Phone number: + 380673056011

E-mail:aks.anastasiia@gmail.com megaly, etc.) [2]. The risk of fetal death depends on gestational age in cases of infection. According to gestational age, maternal infection in the first trimester leads to fetal death in $19 \%$, in $13-20$ weeks $-15 \%$ and after 20 weeks $-6 \%$ of cases [3]. It is quite widespread. Approximately $1-5 \%$ of women is susceptible to parvovirus and develops serologic evidences during pregnancy period; the frequency of morbidity is rising to $3-34 \%$ in epidemic periods; about $50 \%$ of women have an asymptomatic course of the disease [4].

Infection with parvovirus B19 affects many organs and systems, mostly cardiovascular system. The transmission rate of maternal parvovirus B19 infection to fetus is $17 \%$ to $33 \%$ 
[5]. Transplacental viral transmission is probably explained by the presence in villous trophoblastic cells of placental tissues the maximum level of neutral glycolipid known as blood group antigen $\mathrm{P}$ (globoside), which also serves as a receptor for B19, during the first two trimesters of pregnancy. Cardiomyocytes are the most important target cells for parvovirus. P-antigen expressed on fetal cardiac myocytes enables the virus to infect myocardial cells and induce myocarditis that aggravates cardiac failure. Fetal heart failure may be caured by severe anemia but may often be associated with myocarditis, which can cause arrhythmias or even cardiac arrest without evidence of anemia, cardiac failure or hydrops. Infection within myocardium often results in acute inflammation, but may also lead to non-inflammatory damage of cells, and as a consequence, to infection-related cardiomyopathy. Virus B19 enters the endothelium cells utilizing a globoside and its coreceptors a5 pi-integrin and Ku80 and after connection with a receptor pass to the state of persistent infection in the endothelium of various organs, including heart. In consequence, numerous copies of the viral genome of B19 are determined in the endothelium of intramyocardial arterioles, capillaries and post-capillary venules, initiating inflammation process induced by the continuous expression of pro-inflammatory cytokines (TNF-a, interleukins (IL): IL-6, IL-8, IL-2)), IgG, soluble interleukin 2 receptor, leukotrienes and prostaglandins [6]. The direct cytopathic effect of parvovirus, apoptosis, activation of innate and adaptive immune response lead to endothelial dysfunction, followed by ventricular remodeling and development of dilated cardiomyopathy.

The asymptomatic course and late detection of parvovirus B19 infection during antenatal period are two real problems which may lead to the development of cardiac fetal insufficiency.

The advanced ultrasound allows characterizing many complex conditions which are necessary to evaluate and understanding various pathologies that contribute to heart failure in fetus. Doppler ultrasound tools have improved our understanding of fetal circulation and patho-physiological mechanism that controls fetal circulation. According to the literature, ductus venosus (DV) Doppler measurements can give information on pregnancy courses, its prognosis and can therefore be recommended as a part of the routine workup of pregnancies, complicated by viral infections, such as parvovirus B19.
The article describes the methods and results of investigation of blood flow velocity waveforms in fetal DV. These studies are used to visualize the degree of fetal heart failure and determine its further clinical course.

The aim of our research was to predict the development of heart failure in the fetuses that were infected with parvovirus B19 infection during 11-14 gestation weeks by measuring the Doppler parameters of blood flow velocity in the DV.

\section{Methods}

20 pregnant women, who were infected with parvovirus B19 during the period from 11 to 14 weeks of gestation, were involved into the study. The inclusion criteria were: age from 18 to 30 years old; singleton pregnancy; absence of extragenital pathology events in the sub- and decompensation stages; gestation period from 11 to 14 weeks; no episode of a threatened abortion during pregnancy; absence of fetal chromosomal abnormalities. The DV was identified by means of color Doppler. Given that its localization at the cephalic end of intraabdominal portion of umbilical vein we used two approaches in an oblique transverse and sagittal imaging planes through the fetal abdomen to visualize the communication between the umbilical vein and the inferior vena cava. The presence of characteristic high-velocity signals in the color Doppler B-mode proved the identification. Our record was taken in the inlet of DV with an insonation ange as near to the long axis of the vessel as possible. Blood flow patterns merely proved changes in the pressure between the vessel and the right atrium throughout the cardiac cycle. After activating the color Doppler using a low velocity setting $(<0.24 \mathrm{~m} / \mathrm{s})$, three vessels were detected in cross-section: the abdominal aorta, the inferior vena, and the DV. Unlike the superior and inferior vena cavae in which there may be a reverse flow during atrial systole, the flow through the DV proves continuous forward flow towards the heart. Normal flow in the DV was low velocity and triphasic consisting of maximal velocities during ventricular systole (S -wave) in relation with a rapid filling of the atria; the second peak (D-wave) corresponded to the early ventricular diastole, and the flow velocity was minimal during atrial contraction (A-wave). The A-wave directivity evaluation in the DV was investigated according to the Guideline Principles of the Fetal Medicine Foundation (www. fetalmedicine.com). The waveforms were clas- 
sified as normal or abnormal depending on whether the A-wave lowest forward velocity during atrial contraction in late diastole was positive or absent/reversed, respectively. Absent or reverse A-wave in the DV proved fetal cardiac failure. Fetal echocardiography (EchoCG) included assessment of the position of heart, the four-chamber view, the outflow tracts and the venous return to the heart. EchoCG was performed by the ultrasound scanner Philips HD II XE device (USA) using a transabdominal convex probe with the frequency of $3.5 \mathrm{MHz}$, operating in a CDC mode and the frequency filter at $100 \mathrm{~Hz}$. Statistical processing of data was carried out using the package of applied programs Microsoft Office Excel 2016 and Statistica 6, Stata 12. Statistically significant differences at $p<0.05$ were considered.

\section{Results}

Analyzing the results of investigation, in 16 of $20(80 \%)$ fetuses we didn't observe any absent or reversed A-wave flow in the DV during atrial contraction as well as any fetal echocardiographic pathological signs of cardiac damage (Fig. 1). In 2 (10\%) cases the reversed A-wave flow in the DV in a combination with EchoCG-signs of overload of left side of heart, resulting in enlargement (dilatation) of left atrium and left ventricle were detected. In 2 $(10 \%)$ cases the presence of reversed A-wave flow in the DV and EchoCG-signs of fetal heart failure (reduction of cardiac output, significant dilatation of left ventricle) were evidenced (Fig. 2).

In $20 \%$ of the examined women (4 cases) with the presence of reversed A-wave blood

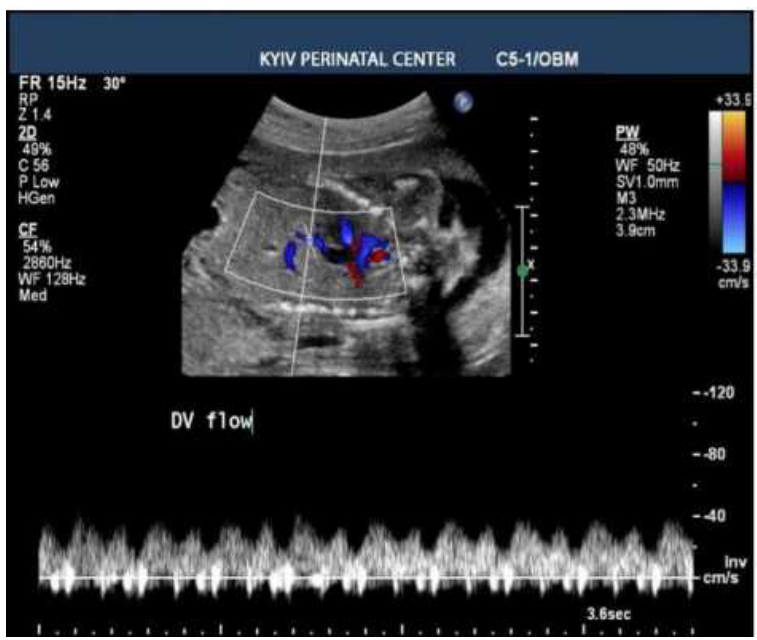

Fig. 1 (leftward). Normal blood flow velocity measured in the ductus venosus during 12 gestation weeks in the woman infected with parvovirus B19 flow velocity in the DV we also made a mathematical correlation between the peak systolic blood flow velocity in the DV and the fetal nuchal translucency thickness, as one of the EchoCG markers of fetal heart failure. The findings confirm that with the expansion of fetal nuchal translucency thickness there is an increased peak systolic blood flow velocity in the DV with the correlation coefficient $r=0.594$ (the coefficient of determination is R2 $=0.3534$ with normal range $r=0.161(R 2=0.0258)$ ) that proves a linear dependence between these two ultrasonography parameters (Fig. 3): the coefficient of determination is the percentage of response variable variation that is explained by a linear model (dependence on the changes of one parameter from another in percentages) - in our case 0,3534 (35,34\%) which means, that the peak systolic blood flow velocity changes in the DV $35.34 \%$ depends on changes in the fetal nuchal translucency thickness and could predict the future fetal cardiac compromise $(p<0.05)$.

\section{Discussion}

Dynamic observation of gestational process allows relate some features of ultrasound pattern evidenced in 11-14-week fetuses with an increased risk of heart failure development. Parvovirus B19 infects primarily the erythroid cell line and may therefore cause fetal anemia and consequently cardiac failure with hydrops fetalis in some cases. Doppler measurement technics today can modify treatment so that the number of fetuses infected with virus B19 and its attendant risks can be minimized, without missing the opportunity for timely, lifesaving intervention when it is warranted.

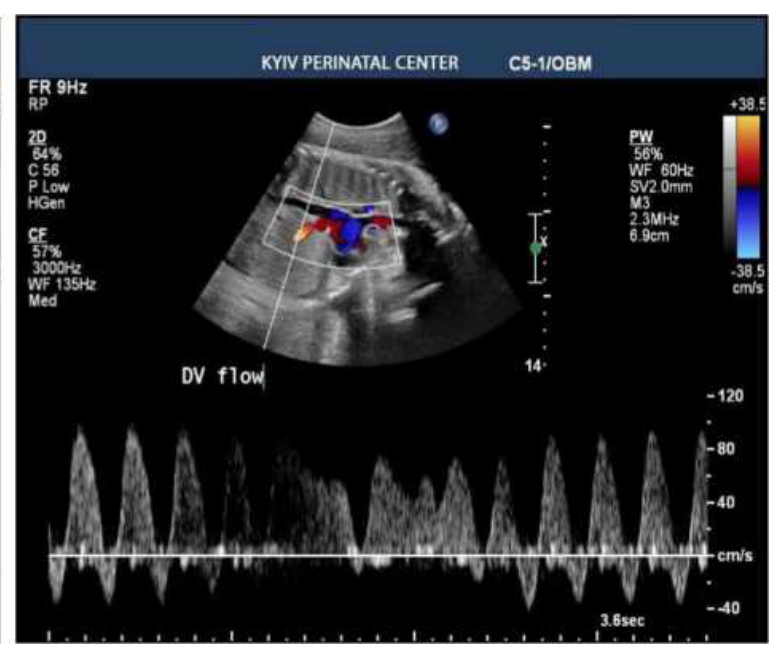

Fig. 2 (rightward). Reversed A-wave flow waveform in the ductus venosus during 12-13 gestation weeks measured in the woman infected with parvovirus B19 


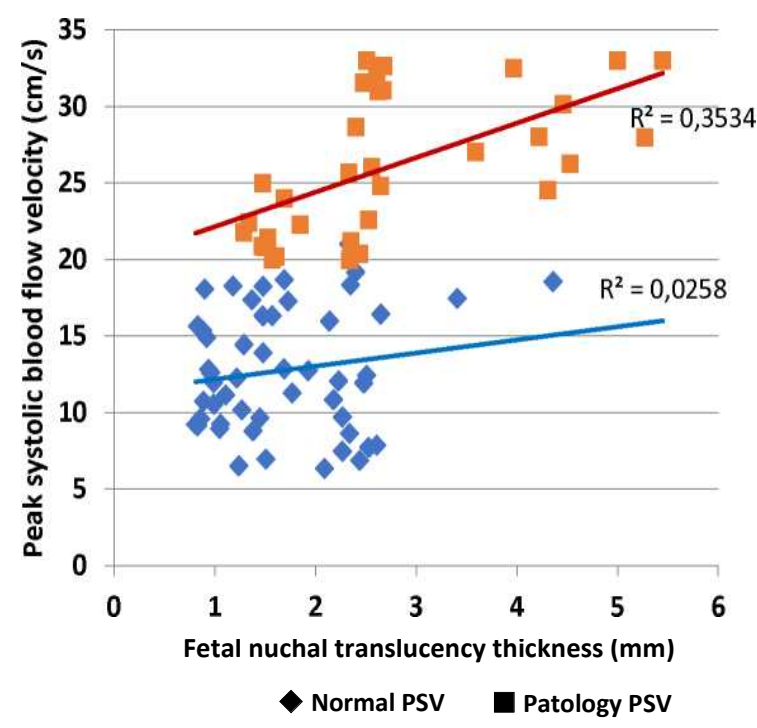

Fig. 3. Correlation curve between the peak systolic blood flow velocity in the ductus venosus $(\mathrm{cm} / \mathrm{s})$ and fetal nuchal translucency thickness (mm)

Increased fetal nuchal translucency thickness was established to be a myocardial dysfunction and/or fetal anemia [7]. The correlation between the increased nuchal translucency and the presence of abnormal blood flow profile in the DV was proved. The measurement of peak systolic blood flow velocity in the DV is the latest tool of non-invasive monitoring of fetuses at risk for anemia and cardiac insufficiency due to parvovirus B19. In the article, the increased fetal nuchal translucency thickness was found with reversed flow in the DV during atrial contraction, this findings were helpful indicators of the presence of fetal heart failure.

\section{References}

1. Arabzadeh S. Human parvovirus B19 in patients with beta thalassemia major from Tehran, Iran. Blood Res. 2017;52(1):50-54.

2. Zavattoni M, Paolucci S, Sarasini A. Diagnostic and prognostic value of molecular and serological investigation of human parvovirus B19 infection during pregnancy. New Microbiol. 2016;39:181-185.

3. Lamont RF. Parvovirus B19 infection in human pregnancy. BJOG. 2011;18(2):175-186.

4. Kerr JR. The role of parvovirus B19 in the pathogenesis of autoimmunity and autoimmune disease. J Clin Pathol. 2016;69(4):279-91.
In summary, the linear correlation between the presence of a reversed A-wave blood flow velocity in the DV and the overload of left fetal heart, the development of heart failure ( $20 \%$ of the total number of examined women) was proved.

\section{Conclusions}

Parvovirus B19 infection has a direct pathological effect on the endothelium of intramyocardial arterioles, capillaries and post-capillary venules. The development of dilated cardiomyopathy and heart failure are two pathological consequences of B19 cardiac damage.

The most reliable way to diagnose fetal heart failure during antenatal period is the Doppler and EchoCG investigation of blood flow velocity waveform in the DV and the measurement of nuchal translucency thickness.

In case of left-sided heart insufficiency occur the volume overload of right side of fetal heart with increased pressure in the DV, the presence of a reversed A-wave flow velocity in the DV and increased peak systolic blood flow, the expansion of fetal nuchal translucency thickness and decreasing left ventricular isovolumic relaxation time.

A linear relationship between the increased fetal nuchal translucency thickness and the peak systolic blood flow velocity in the DV was determined.

According to the results of the study, we can conclude that there is a direct correlation between the appearance of a reversed A-wave blood flow in the DV and the development of fetal heart failure.

5. Crane J. Parvovirus B19 Infection in Pregnancy. J Obstet Gynaecol. 2014;36(12):1107-1116.

6. VerdonschotJ. Relevance of cardiac parvovirus B19 in myocarditis and dilated cardiomyopathy: review of the literature. European Journal of Heart Failure. 2016;18,1430-1441.

7. Hichijo A, Morine M. A case of fetal parvovirus B19 myocarditis that caused terminal heart failure. Case Reports in Obstet and Gynecol. 2014:1-4.

Received: 2017-10-19 